\title{
The Design of Digital Filter System used in Stimulation with Tomatis Method
}

\author{
Krzysztof Jóźwiak \\ Lodz University of Technology \\ Institute of Electronics \\ ul. Wólczańska 211/215, 90-924 Łódź, Poland \\ Email: krzysztof.jozwiak@edu.p.lodz.pl
}

\author{
Michał Bujacz, Aleksandra Królak \\ Lodz University of Technology \\ Institute of Electronics \\ ul. Wólczańska 211/215, 90-924 Łódź, Poland \\ Email: \{aleksandra.krolak, michal.bujacz\}@p.lodz.pl
}

\begin{abstract}
The Tomatis Method is a rehabilitation technique used in psychology, the main aim of which is stimulating the cochlea in the inner ear by filtered air-conducted and boneconducted sounds. The system of electronic filters and amplifiers used for this therapy is called the Electronic Ear. Commonly, it is an analog device, that is expensive and after a few years its functionality declines. In this paper, we introduce a digital Electronic Ear system using an STM32F4 family micro-controller and ADC/DAC integrated circuits. The design of digital sound filters allows to adjust more parameters and overcomes some of the constraints of analog systems. In this paper, we provide a short review of the Tomatis Method, the main functions of the Electronic Ear and we describe the designed system with comparison measurements to the analog one.
\end{abstract}

\section{INTRODUCTION}

$\mathbf{M}$ USIC therapy has numerous applications in psychology and rehabilitation. It can be used to help patients manage stress, concentration or depression. However, in many cases these methods do not give visible effects, when they are compared with a control group [1-4].

The Tomatis Method (TM) has been shown to help people with various psychological disorders (autism, dyslexia, ADD and more) [10], [11], [12], [13], [14], [15], [16], [17], [18], [20] though in some controlled trials the results were also inconclusive [8],[19]. It uses music, but it is not a music therapy, as it is based on brain and inner ear stimulation by selective sound filtering. The electronic device that implements the filter system for TM is called an Electronic Ear (EE). Most EEs that are commonly used are analog systems, that have their own constraints and high prices. We propose a new, digital Electronic Ear (DEE) system designed for cheaper implementation of TM and its further studies.

\section{TOMATIS AUdio-Psycho-Phonology}

Tomatis Laws cover the theory behind the relationship of hearing and speaking. Alfred Tomatis observed that problems with singing or speaking in some particular spectrum are connected with difficulties with hearing in the same spectrum and proposed that the improvement of vocal and voice range can be provided by opening the hearing ability to a specific frequency spectrum. This principle is called the Tomatis effect. Over the years his ideas were implemented in other forms of sound therapy and a generic Electronic Ear tool was developed.
The Electronic Ear is based on the concept of stimulating the basilar membrane (BM) in the cochlea in two ways by air and bone conduction. Air conduction is based on the typical ear pathway, with sound vibrations picked up by the eardrum, transferred by the ossicles to the oval window of the cochlea. Bone conduction is the perception of vibrations travelling through the bone and stimulating the BM either through pressure changes in the cochlear fluid or vibrations of the cochlear wall [5]. Bone conducted sounds reach the brain faster due to the higher density of the medium; however, large part of the acoustic signal is reflected due to the air-bone impedance mismatch. That is why bone conduction mostly favors our own voice. The conclusion drawn by Tomatis from this observation was that air conduction is used to "communicate with the outer world" and bone conduction is used to "listen to oneself". [6],[7].

The next feature that is important for TM is human earedness, i.e. which ear is dominant in the hearing process. Left ear dominance is problematic, because the left ear has the longer path to the left hemisphere. The delay between left and right ear is about 0.4-0.9 ms. People, who are left-eared have a lot of listening problems that can cause various psychological disease, such as dyslexia [6]. One of the main aims of Tomatis Rehabilitation is to increase the role of the right ear for the people who are left-eared. For this reason there are separate delays and adjustable volume levels for left and right channels.

Fig. 1 shows the flow chart of the EE. It is an audio system, the main function of which is amplifying, gating and filtering sound going to the three output channels - air left, air right and bone conduction. Gating is the process of changing the filter type depending on signal level. Below a certain amplitude level the $\mathrm{C} 1$ filter is on and above a certain level the filter is switched to $\mathrm{C} 2$ filter. The sudden change gives an impulse that theoretically stimulates the brain. The $\mathrm{C} 1$ and $\mathrm{C} 2$ filters are shelving filters with $1 \mathrm{kHz}$ crossband frequency. The boost/attenuation level is adjusted by the specialist administering the TM therapy and can be set from -5 to $5 \mathrm{~dB}$. The EE also contains a second filter - a High Pass filter with an adjustable cut-off frequency. It is supposed function is to imitate the medium of a mother's womb. In some cases there is a need to increase or decrease a role of bone conduction in comparison to air conduction. For this reason 


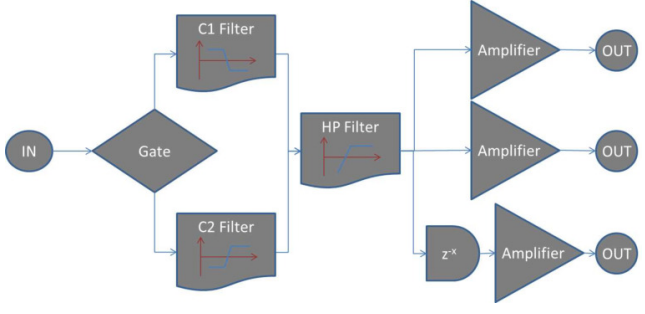

Fig. 1. Flow chart of the Electronic Ear (EE). The Gate depending on the signal level diverts it to the shelving filters $\mathrm{C} 1$ or $\mathrm{C} 2$. The HP filter cuts off low frequencies. The Delay allows to the air and bone conducted sounds to arrive at different times and the Amplifiers control the level of the three outputs.

there is an adjustable delay between the air and bone channel called precession.

TM is used for many purposes. One of the popular aims is in therapy of autistic children. According to Tomatis theory autism is connected with problems with listening to the external world - in most autistic children bone conduction sensitivity is higher than air conduction. The therapy's aim is to lower this sensitivity in order to open the child up to communication with others. There were many studies carried out to verify the effectiveness of this rehabilitation. One of the most controversial was [8]. It revealed that there was no clear difference between the effects on a control group and an experimental group put under TM rehabilitation. For response to this article there were two follow-up studies [9], [10]. They showed the opposite view and pointed out missteps in the previous research. These two and another one [11] show a significant improvement after the TM rehabilitation measured in the Children's Autism Rating Scale (CARS) or in Gilliam Autism Rating Scale (GARS).

An overview of over 30 TM studies by Gerritsen showed that the majority of studies demonstrated positive effects of TM therapy. Some of the better documented TM treatments include:

- reduction of the Attention Deficit Disorder (ADD) by, similarly to autism, increasing the focus on air conducted sounds versus bone conduction [6],[12],[13].

- treating dyslexia [6],[13],[20] (that is theoretically connected with left-earedness) by increasing the sensitivity of the right ear.

- supporting language learning [14], by setting the $\mathrm{C} 1 / \mathrm{C} 2$ filters to frequencies most commonly appearing in a given language. TM rehabilitation has also been successfully used for:

- Epilepsy - visible effect in more than 50

- Cerebral palsy: significant improvement [16]

- Stuttering and hoarseness [13]

- Emotional problems including depression: high effect, compared with a control group [17]

- Music skills improvement: results are divided [18],[19]

Most of tests have shown a positive effect of TM, but there are also those, that did not report any differences when compared to a placebo treatment. We need to consider that negative re- sults can stem from inappropriateness in investigation methods or in the way the EE device was used [9]. This shows that the $\mathrm{TM}$ requires further rigorous studies.

\section{ELECTRONIC EAR STIMULATOR}

In terms of the electronic design main functions of Electronic Ear are: Converting audio signal to digital data (ADC); Pre-processing; Gating; Filtering; Digital-Analog converting (DAC); Amplifying. The device can be divided into four main modules: audio input, audio output, micro-controller and power supply. Three voltage levels are needed:

- 5V - external power supply. It is used as an input for the step-down converter and as an analog power supply for the input section. The transformer power source is used in order to decrease the distortions in the low frequency audio signals.

- $3.3 \mathrm{~V}$ - it is given by Low Dropout Positive Regulator TC2117 from MicroChip. It is used for digital power supply for $\mu \mathrm{C}$ and for the digital part (communication interfaces) of the ADC/ DAC converters.

- $2.5 \mathrm{~V}$ - it is necessary for the output analog power supply and it is provided by LP2985-N

In this project external 24-bit stand-alone integrated circuits were used. For input a PCM4201 from Texas Instruments and for output CS432L22 from Cirrus Logic were used. In both cases communication between the audio converter and $\mu \mathrm{C}$ was provided by an I2S interface, the audio data word length is 24 bits and is Left-Justified in the frame. CS43L22 supports I2S and it was working in the slave mode by being connected to four pins: MCLK, LRCK, SCLK and SDO. It has its own transmission interface that has 3 communication ports - BCK (equivalent to SCLK), DATA (equivalent to SDO), FSYNC (equivalent to LRCK) and one system clock port, that is necessary for proper work. The serial interface is very similar to I2S and after a few modifications it can work in high performance mode communicating in master mode. The main difference is that the system clock needs to be 512 times larger than the sampling frequency (256 times larger in I2S mode). To get that result an extra I $2 \mathrm{~S}$ interface was used with a 2 times larger sampling frequency and the MCLK pin connected to the system clock pin in the audio converter. That allows a clean transmission without errors.

The CS43L22 is a DAC converter, but also a headphone amplifier. It allows to prevent from using two independent chips (one for converting, one for amplifying) and minimalizes space on the PCB board. In the project there are two output channels - air and bone channel. In order to support three channels (that are required for the EE) there is used an external adapter that splits the air channel to left and right channels with a build-in balance regulator. CS43L22 has two serial interfaces and except of I2S for data transmission it has also $\mathrm{I} 2 \mathrm{C}$ interface for control. It allows to regulate all functions of the chip, for example volume, that is continuously sent to the control register.

For digital signal processing the STM32F407VGT6 microcontroller was used. Data was stored in a float buffer. Its 
size was set in order to provide maximum 500 milliseconds delay, which for $48 \mathrm{kHz}$ frequency sample gave $(24000+$ filter order) size. User interface gives possibility to adjust the main parameters of the $\mathrm{EE}-\mathrm{C} 1, \mathrm{C} 2$ levels value, frequency, precession and volume and the advanced options as gate upper level and four options for filter order. Changing filter order affects its selectivity and as a result the intensity of stimulation.

There were used filter orders in range of 51 to 101 (only odd orders are used). Every filter was designed as FIR (Finite Impulse Response) filter using the window method. The window that was chosen for this purpose was modified Tukey window (tapered cosine window). It is combination of a rectangular window and a Hamming window with an $\alpha$ coefficient that determines what part of the window should be flat. It makes cosine lobe of width $\alpha / 2 * \mathrm{~N}$ ( $\mathrm{N}$ is filter order) and rectangular window of width $(1-\alpha / 2) * \mathrm{~N}$ at the center of filter function. With $\alpha=0.8$ it gives the best results - sufficient filter selectivity providing expected filter band levels even for low cross-frequency (500 Hz is a minimum used value) and low ripple frequency response.

In this project two types of filters are required - simple HP/LP filter and a shelving filter for the $\mathrm{C} 1$ and $\mathrm{C} 2$ filtering. To obtain a two-level shelving filter it was necessary to use a parallel connection of filters - one for left part (LP filter) and one for right part (HP filter). The cross-frequency is set where the characteristic crosses the $0 \mathrm{~dB}$ line. For that reason there was a need to modify one frequency by a coefficient dependent on filter order and $\mathrm{C} 1$ and $\mathrm{C} 2$ levels.

The big challenge was to appropriately design the filter in case when both shelving filter levels have the same sign. Normally it should not cross the zero decibels line. But measurements of the original EE show, that with this case it should be artificially modified in order to give $0 \mathrm{~dB}$ gain at the cross-frequency point. A cascade connection of two filters with similar cut-off frequency was used, but modified regarding to the purpose. For the positive shelving filter levels the gain near the cross-frequency was decreased (frequencies of filters diverge) and for negative it was increased (frequencies converge). Then, obtained filter is serially connected with the normal shelving filter.

In this section the frequency-responses of filters in the designed DEE are showed and compared with those measured for the original, analog EE device.

Fig. 2 and 3 show frequency-responses for 101-order filters for designed device. As it can be seen, passband levels have correct values, remain stable and $\mathrm{C} 1$ and $\mathrm{C} 2$ filter lines cross near the $0 \mathrm{~dB}$ level. In the first case the $500 \mathrm{~Hz}$ (the minimal needed value for this project) and in the second $1 \mathrm{kHz}$ (frequency of analog EE) cross-band frequency was set. In figure 3 the filter with two positive levels was shown to provide appropriate gain modification near cross-frequency.

\section{Results}

In Fig. 4 and 5 frequency responses measured for the DEE and analog $\mathrm{EE}$ are shown for $1 \mathrm{kHz}$ cross-band frequency and $[ \pm 5, \pm 5]$ and $[ \pm 5, \pm 1]$ levels respectively. The unstable

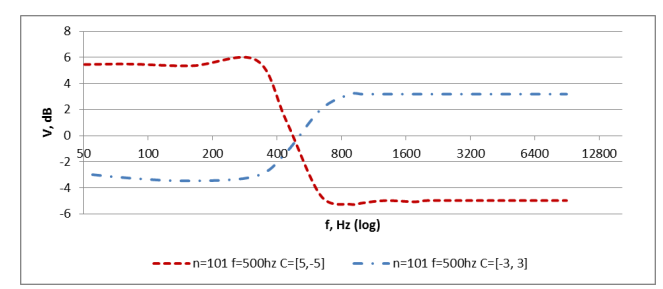

Fig. 2. Frequency-response for $\mathrm{n}=101, \mathrm{f}=500 \mathrm{~Hz}$ and passband levels set.

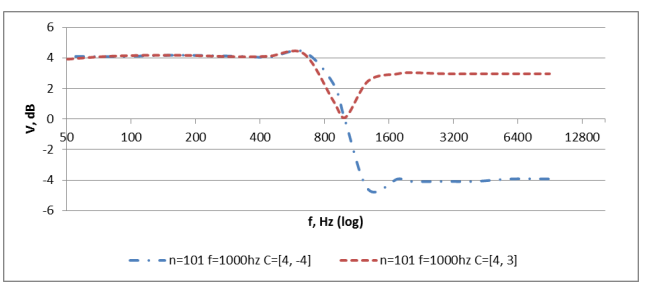

Fig. 3. Frequency-response for $\mathrm{n}=101, \mathrm{f}=1000 \mathrm{~Hz}$ and passband levels set.

and exceeded passband levels during the whole frequency bandwidth for analog devices can be seen. In Fig. 5 one can notice the ringing artifacts for the bandwidth above $1 \mathrm{kHz}$. The cross-point for analog device also has an incorrect value i.e. $850 \mathrm{~Hz}$ for first case and $865 \mathrm{~Hz}$ for the second case.

\section{Conclusions}

The DEE, which was the topic of this paper was designed properly and it provides all the required functions. It services 2 channels, that with external splitting adapter allow to support three channels. If the right/left channel delay was needed, there would be a possibility to use second, same DAC integrated device for extra output channels. The DEE uses 24-bit audio data with $48 \mathrm{kHz}$ sampling frequency. The included filters give the desired effects - the shelving filter levels and crossfrequency value are as expected. It monitors changes at air and bone channels and gates them to $\mathrm{C} 1$ or $\mathrm{C} 2$ filters depending on the signal level and precession value.

Compared to the analog EE, the designed device has all the same functions, but it has also extra - features allowing to remove some previous constraints. First it is a digital system, so the filter parameters are stable and do not depend on

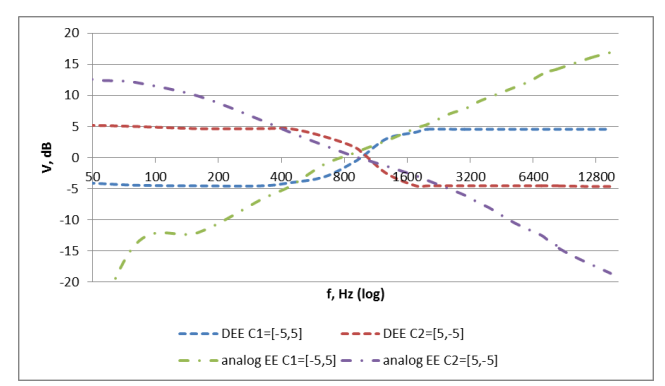

Fig. 4. Frequency-response of $\mathrm{C} 1 / \mathrm{C} 2$ filters measured for digital and analog device. Theoretically, the analog EE's pass bands should be flat, but the measurements show they clearly have a linear drop-off. 


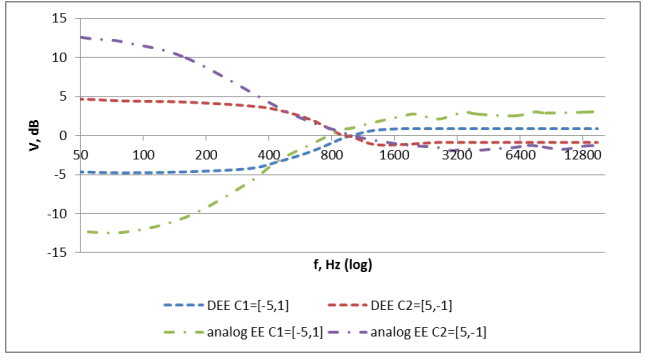

Fig. 5. Frequency-response of $\mathrm{C} 1 / \mathrm{C} 2$ filters measured for the proposed digital and the original analog TM device.

the duration of operation or component aging. In Figures 4 and 5 there is visible effect of this occurrence for the analog device - the filter levels are different than expected and are unstable. We can only speculate about the discrepancies between the theoretical spectra and those measured for the commercial EE analog device. One theory is component aging (e.g. degradation of dielectric in the capacitors) or it could simply be a design flaw. Another advantage is that the cost of production of the DEE (less than 200EUR) is much lower than the analog TM device (approximately 10,000EUR). The fact that filters in the designed system are digital also gives an opportunity to develop it in the future, change the parameters or to upgrade it depending on the requirements.

The next advantage is that digitalization allows more parameters to be adjusted by the user. It is possible to set the cross-frequency of the shelving filter. In analog EE C1 and C2 filters have always the same cross-frequency and it is $1 \mathrm{kHz}$ It has also only one type of filter selectivity. In the designed device four cases for filter order allow to change the intensity of stimulation and pick the best one for the individual patient. The changeable filter frequency allows to modify frequency band, that impacts the most to the listener.

To sum up, a modernized digital version of the device used for the rehabilitation by the Tomatis method was designed and tested. Its performance was measured to be the same or better than the original equipment, while allowing much more control over the functionality.

\section{REFERENCES}

[1] L. Hohmann, J. Bradt, T. Stegemann, S. Koelsch, "Effects of music therapy and music-based interventions in the treatment of substance use disorders: A systematic review," PLOS, 2017, doi: 10.1371/journal.pone. 0187363 .
[2] M. Bodner, R. P. Turner, J. Schwacke, C. Bowers, C. Norment, "Reduction of Seizure Occurrence from Exposure to Auditory Stimulation in Individuals with Neurological Handicaps: A Randomized Controlled Trial," PLOS, 2012, doi: 10.1371/journal.pone.0045303.

[3] M. Bucur, A. L. Marian, "The impact of the Mozart effect on creativity myth or reality," Creativity, 2016.

[4] L. C. Lin, W. T. Lee, H. C. Wu, C. L. Tsai, R. C. Wei, H. K. Mok, "The long-term effect of listening to Mozart K.448 decreases epileptiform discharges in children with epilepsy," Epilepsy Behav. vol. 21(4), 2011, pp. 420-424, doi: 10.1016/j.yebeh.2011.05.015.

[5] P. Henry, T. Letowski, Bone conduction: Anatomy, physiology, and communication, Army Research Laboratory, 2007.

[6] P. Sollier, Listening for wellness: An Introduction to the Tomatis Method, The Mozart Center Press, 2005.

[7] N. Doige, A bridge of sound in: The brain's way of healing, Ed. Mińska Barbara, Vital, 2016.

[8] B. A. Corbett, K. Shickman, E. Ferrer, "Brief Report: The Effects of Tomatis Sound Therapy on Language in Children with Autism," $J$. Autism Dev Disord., vol. 38(3), 2007, pp. 562-566, doi: 10.1007/s10803007-0413-1

[9] J. Gerritsen, "Response to Brief Report: The Effects of Tomatis Sound Therapy on Language in Children with Autism," J. Autism Dev Disord., vol. 38(3), 2008, pp. 567, doi: 10.1007/s10803-007-0471-4

[10] M. AbediKoupaeia, K. Poushanehb, A. Z. Mohammadic, N. Siampour, "Sound Therapy: an Experimental Study with Autistic Children," Procedia - Social and Behav. Sciences, vol. 84, 2013, pp. 626-630

[11] J. M. Neysmith-Roy, "The Tomatis Method with severely autistic boys: Individual case studies of behavioral changes," South African J. Of Psychology, vol. 31(1), 2001.

[12] L. Sacarin, Early Effects of the Tomatis Listening Method in Children with Attention Deficit, AURA - Antioch University Repository and Archive, 2013.

[13] J. Gerritsen, A Review of research done on Tomatis Auditory Stimulation, Mozart Center, 2009.

[14] I. M. du Toit, W. F. du Plessis, D. K. Kirsten, "Tomatis Method Stimulation: Effects on Student Educational Interpreters," J. of Psych in Africa, vol. 21(2), 2011, doi: 10.1080/14330237.2011.10820454.

[15] G. Coppola, A. Toro, F. F. Operto, G. Ferrarioli, S. Pisano, A. Viggiano et al., "Mozart's music in children with drug refractory epileptic encephalopathies," Epilepsy Behav., vol. 50, 2015, pp. 18-22, doi: 10.1016/j.yebeh.2015.05.038.

[16] I. Przybek-Czuchrowska, E. Mojs, E. Urna-Bzdęga, ”Opis przypadku dziecka z organicznym uszkodzeniem w obrębie ośrodkowego układu nerwowego leczonego metoda treningu słuchowego Tomatisa," Neuropsychiatria i Neuropsychologia, vol. 10(1), 2015, pp. 40-45.

[17] J. O. Coetzee, The effect of the Tomatis Method on depressed young adults, Potchefstroom University, 2001.

[18] W. du Plessis, S. Burger, M. Munro, D. Wissing, W. Nel, "Multimodal enhancement of culturally diverse, young adult musicians: a pilot study involving the Tomatis method," South African J. of Psych., vol. 31(3), 2001

[19] A. Vercueil, H. Taljaard, W. du Plessis, "The effect of the tomatis method on the psychological well-being and piano performance of student pianists: an exploratory study," SAMUS, vol. 31, 2011.

[20] T. Gilmor, "The efficacy of the Tomatis method for children with learning and communication disorders: A meta-analysis," Int. J. of Listening, vol. 13(1), 1999, doi: 10.1080/10904018.1999.10499024. 